\title{
Monitoring dynamic spatial fields using responsive geosensor networks
}

\author{
Matt Duckham \\ Department of Geomatics, \\ University of Melbourne, \\ Australia \\ mduckham@unimelb.edu.au \\ Silvia Nittel \\ NCGIA, University of Maine \\ Orono, ME 04473, USA \\ nittel@spatial.maine.edu
}

\author{
Mike Worboys \\ NCGIA, University of Maine \\ Orono, ME 04473, USA \\ worboys@spatial.maine.edu
}

\begin{abstract}
Information about dynamic spatial fields, such as temperature, windspeed, or the concentration of gas pollutant in the air, is important for many environmental applications. At the same time, the development of geosensor networks (wirelessly communicating, sensor-enabled small computing devices, distributed throughout a geographic environment) present new opportunities for monitoring of dynamic spatial fields in much more detail than ever before. This paper presents a new model for querying information about dynamic spatial fields using geosensor networks. In order to manage the inherent complexity of dynamic geographic phenomena, our approach in this paper is to focus on the qualitative representation of spatial entities, like regions, boundaries, and holes, and of events, like splitting, merging, appearance, and disappearance events. Based on combinatorial maps, we present a qualitative model as the underlying data management paradigm for geosensor networks that is capable of tracking salient changes in the network in a much more energy-efficient way. Further, our model enables reconfiguration of the communication in the geosensor network in response to changes in the environment. We present an algorithm capable of adapting sensor network granularity according to dynamic monitoring requirements. Regions of high variability can trigger increases in the geosensor network granularity, leading to more detailed information about the dynamic field. Conversely, regions of stability can trigger a coarsening of the sensor network, leading to efficiency increases in particular with respect to power consumption and longevity of the sensor nodes. Querying of this responsive geosensor network is also considered, and the paper concludes with a review of future research directions.
\end{abstract}

\section{INTRODUCTION}

Sensor networks are distributed ad-hoc wireless networks of sensor-enabled miniature computing platforms. A geosensor network is defined as a sensor network that monitors

Permission to make digital or hard copies of all or part of this work for personal or classroom use is granted without fee provided that copies are not made or distributed for profit or commercial advantage and that copies bear this notice and the full citation on the first page. To copy otherwise, to republish, to post on servers or to redistribute to lists, requires prior specific permission and/or a fee.

GIS'05, November 4-5, 2005, Berlin, Germany

Copyright 2005 ACM 1-58113-979-9/04/0011 ...\$5.00. phenomena in geographic space [19]. This paper presents a new model for monitoring and querying dynamic information about the geographic environment using geosensor networks. Geosensor networks present important new opportunities to a wide range of environmental applications.

As an example, in June 1991 Mount Pinatubo on the island of Luzon in the Phillipines erupted catastrophically after about 600 years of dormancy. Figure 1 shows a photograph following the eruption in 1991. Layers of ash surround the crater and the effect of mud-flows in this previously heavily forested and agricultural region can be traced as dark ribbons flowing downhill to the north west of the crater. In such a scenario, emergency response managers are interested in whether the mud flow is still expanding; whether one of the major tributaries has split; whether a hole has developed in the flow; and the position of the major boundaries. The provision of such information requires the ability to monitor and represent qualitative information about a dynamic spatial field, in this case the mud flow.

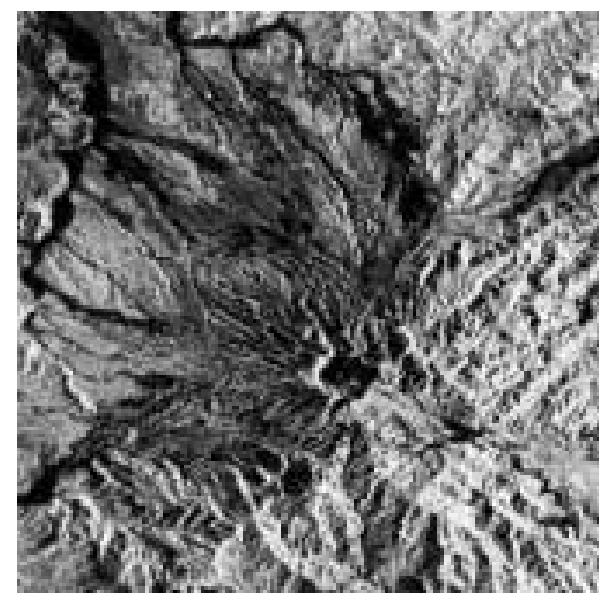

Figure 1: Mount Pinatubo following eruption in 1991 (mudflows shown as darker areas north and west of volcano). Source: NASA/JPL.

In this paper, we build the theoretical foundations for organizing and querying geosensor networks with application to the monitoring of dynamic environmental phenomena, such as mud flows, ash clouds, and gas concentrations. The primary contributions of this paper are:

- a qualitative model for data management in geosensor 
networks to monitoring dynamic spatial phenomena based on combinatorial maps;

- an algorithm for building and maintaining an adaptive network topology for geosensor network which responds to changes in the monitored geographic environment; and

- an approach to in-network query processing for deriving qualitative information about complex dynamic spatial phenomena.

\section{DYNAMIC FIELDS}

Research into spatiotemporal information systems may be divided into work on movement/change to geographic objects, and movement/change to dynamic fields. Examples of the former include work on moving object databases (e.g., [25]), mobile ad-hoc networks (e.g., [18]), and locationbased services (e.g., [11]). Past research into dynamic spatial fields, also sometimes called continuous phenomena, has concentrated primarily on modeling issues. The integration of predictive dynamic field-based modeling capabilities into GIS has been a particular focus in topics such as hydrology and natural resource management (e.g., $[1,12,17])$. Peuquet [21] outlines some of the issues surrounding modeling changes in dynamic fields from a GIS perspective. Raper and Livingstone [22] discuss the development of information system designed to store and process dynamic field-based data. Such research highlights the importance of identifying salient entities from underlying dynamic fields, such as spits and sand bars in coastal geomorphology $[13,22]$.

In this paper, we focus specifically on changes in dynamic spatial fields. The goal is the observation, monitoring and analysis of environmental phenomena such as wildfires, flooding, detection and tracking of toxic spills or other types of environmental contamination, or monitoring the level of pesticides.

\subsection{Definition of dynamic fields}

A spatial scalar field represents the variation of some scalar property over a region of space. Examples of the scalar properties include temperature, wind-speed, or the concentration of a gas pollutant in the air. A spatial field is defined as a function from space to a scalar property. Formally, given a spatial framework $S$ and some class of scalar values $V$, a spatial scalar field is a function whose domain is $S$ and codomain is $V$. In this paper we will be specifically considering spatial frameworks which are orientable surfaces (see section 3).

The focus of this paper is fields that can change through time. To this end, we define a dynamic spatial scalar field as a function $f$ from a temporal domain $T$ to a spatial field $S \rightarrow V, f: T \rightarrow S \rightarrow V$. The temporal domain $T$ may be consist of instants or intervals, and may be linearly or partially ordered, depending on the set-up of the particular application. From now on all fields will be assumed to be spatial and scalar, so we shall refer simply to "dynamic fields."

\subsection{Qualitative aspects of dynamic fields}

There are at least three important modeling and computational advantages to studying the qualitative (rather than quantitative) aspects of space. After Galton [6]:
- Qualitative properties form a small, discrete domain, whereas quantitative properties form a large, continuous domain, often modeled by real numbers.

- Qualitative properties are supervenient on, and derivable from, quantitative properties.

- The boundaries between qualities correspond to salient discontinuities in our apprehension of quantitative properties.

These general advantages translate into three specific advantages of adopting a qualitative approach to dynamic fields in this paper, in particular with respect to environmental monitoring using geosensor networks.

First, due to the relative size of the domains, processing and communication with qualitative information potentially enables more efficient use of limited computational, communication and energy capabilities. Today, computational and communication capabilities are the major limiting factors for the use of geosensor networks in environmental monitoring, and need to be used efficiently to maximize application lifetime of a sensor network.

Second, while it is always possible to generate qualitative representations of quantitative phenomena, the converse is not true. Thus, purely qualitative information, such as the knowledge that two geosensor nodes are in close proximity because they can "hear" each other's communication signal, cannot be meaningfully transformed into qualitative information about the precise distance between the geosensor nodes. For example, a distant sensor node might emit a strong signal because of the use of an amplifier, thus, appear closer than a collocated sensor node with a weak signal. To take advantage of such neighborhood information, a qualitative representation and reasoning system is required.

Third, dynamic fields are highly complex phenomena. Managing the complexity of information about dynamic fields is often approached in a quantitative approach, thus, attribute values are estimated using spatial data analysis methods that can lead to a coarse estimation, and are computationally expensive [23]. In contrast, we propose the use of a qualitative approach, to construct salient entities from complex dynamic fields. This in turn will ensure that information systems for dynamic fields are easier for designers to construct and for users to query as well as more energyefficient to track and compute. Two types of emergent entities result from a qualitative approach to dynamic fields, namely continuants that endure through time (e.g., regions, boundaries, holes) and occurrents that happen or occur and are then gone (e.g., splitting events, appearance of holes, disappearance or merging of regions). Galton [7] makes the distinction between histories that are functions from a temporal domain to attribute values, or properties of objects, and chronicles that treat dynamic phenomena as collections of happenings. Grenon and Smith [8] call temporal sequences of continuant configurations the SNAP ontology, and the event/process view, the SPAN ontology.

One of the primary objectives of this paper is to develop a qualitative approach to modeling dynamic fields in a way that is compatible with the identification of emergent continuants and occurrents. The ultimate aim is to enable the development of simple tools for designing and querying geosensor networks for environmental monitoring of dynamic fields. 

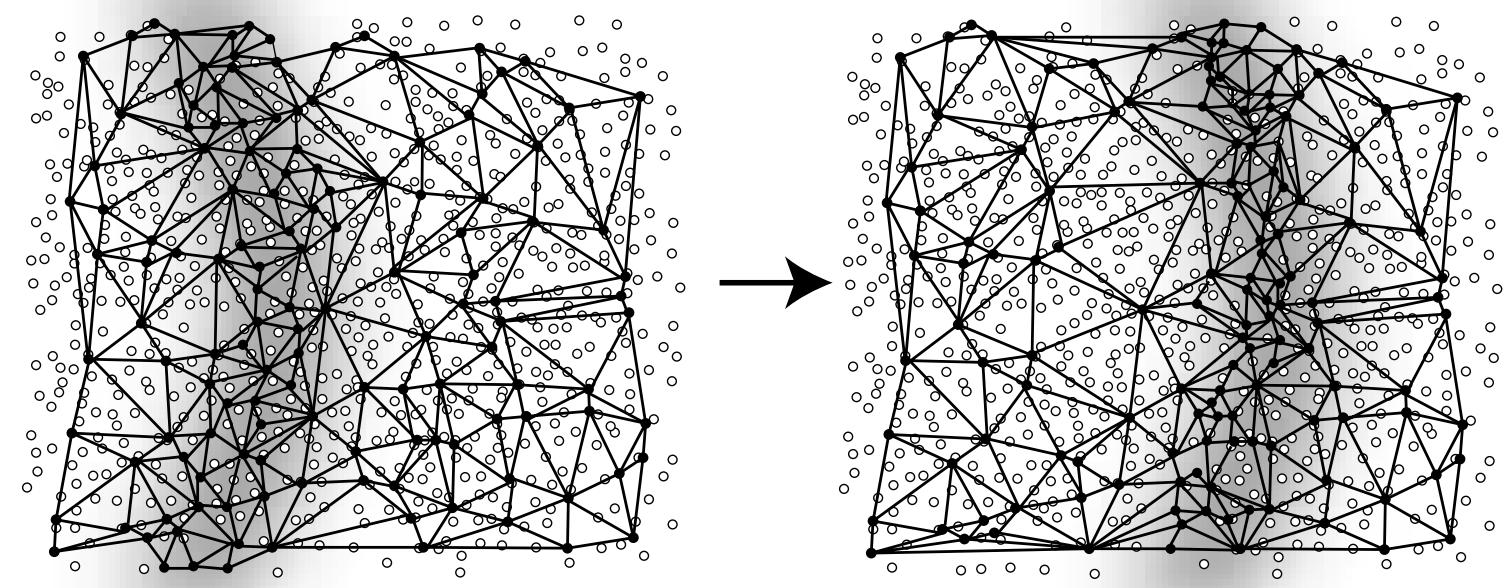

Figure 2: Configuration of sensors and triangulation in response to movement of a front

\section{SENSORS \& COMBINATORIAL MAPS}

At the foundation of our approach is a formal model of sensor networks based on combinatorial maps.

\subsection{Geometric framework: Triangulations}

Data associated with the dynamic field is collected by a network of sensors, assumed to be fixed, point-based devices, finite in number, and embedded in a surface. Let $S$ denote the surface under consideration (a mathematical abstraction of a portion of the Earth's surface, and assumed to be orientable), and let $P \subseteq S$ be denote the finite set of points on the surface at which the sensors are positioned. The point set $P$ gives rise to a collection of connectivity structures that also provide tessellations of the surface, where the vertex sets of the structure are subsets of $P$. The idea is that at any time, the specific subset of $P$ under consideration will represent the set of locations of currently active sensors. The tessellations used in this work are triangulations, as they are topologically simple and provide efficient frameworks for interpolation of field values.

The fact that the vertex set is allowed to be a proper subset of $P$ is a critical part of this approach, as we assume that at any one time only a small proportion of the sensors are turned on, and it is this proportion that will be measuring values of the field at that time. Figure 2 shows the approach schematically. The set $P$ is indicated by the collection of small circles; the black circles indicate the sensors that are currently turned on; the unfilled circles indicate sensors that are currently in sleep mode; a triangulation has been formed using as nodes the sensors that are currently active. Figure 2 also shows the requirement for the collection of active sensors and corresponding combinatorial map to be responsive to change. In the figure, we see the movement from left to right over time of a front, maybe the boundary of a region of high field activity, and the change in the spatial distribu- tion of sensors in response to this movement (higher sensor density being required at the critical boundary region).

\subsection{Topological framework: combinatorial maps}

While the triangulation provides the appropriate communication and data processing geometry for the sensor network, there is also a requirement for a topological structure, so that the network can recognize qualitative and topological changes, such as splitting of a region, or development of a hole. The discrete structure we use to formally model this tessellation is the combinatorial map. The combinatorial map was first introduced by Edmunds [5] and developed by Guibas and Stolfi [9] and others (e.g., [4]).

Definition: A (2-dimensional) oriented combinatorial map, or just map, $\mathcal{M}$, is a triple $\left\langle D, \alpha_{0}, \alpha_{1}\right\rangle$, where $D$ is a finite set of elements, called darts, $\alpha_{0}$ is an involutory bijection on $D$ (i.e., $\alpha_{0}^{2}=1$ ), and $\alpha_{1}$ is a bijection on $D$.

Definition: Let $\mathcal{M}=\left\langle D, \alpha_{0}, \alpha_{1}\right\rangle$ be a map. A submap of $\mathcal{M}$ is a triple $\mathcal{M}^{\prime}=\left\langle D^{\prime}, \alpha_{0}^{\prime}, \alpha_{1}^{\prime}\right\rangle$, which is itself a map, and where $D^{\prime} \subseteq D$, and $\alpha_{0}^{\prime}$ and $\alpha_{1}^{\prime}$ are restrictions of $\alpha_{0}$ and $\alpha_{1}$, respectively.

These definitions are extended to accommodate qualitative properties of sensor nodes and sensor node-node relations. In terms of the sensor application, the map $\mathcal{M}$ provides a model of the configuration of all sensors in the surface, and their potential communication partners.

Each dart $d$ represents a capability of a sensor for a potential communication partner within its communication range, and the dart $\alpha_{0} d$ represents the capability of its partner. The function $\alpha_{1}$ gives rise to a permutation that can be used to represent the sensor nodes themselves. Each permutation 
contains a cyclic ordering of darts. This ordering provides the qualitative directions from a node to each nearby sensors.

We need to provide more information than provided by the pure combinatorial map in order to make reasonable decisions about actual communication partners in real time. With each dart is stored a qualitative distance/reliability indicator (e.g., close, midrange, far). Figure 3 shows schematically the geometric-topological information associated with each sensor, as it is located in the network. The sensor node itself is represented as the cyclic permutation $(a, d, e, c, j, g, f, h, i, b)$ of darts $\{a, b, c, d, e, f, g, h, i, j\}$, giving the cyclic ordering of links around the sensor, with this information also providing the relative qualitative directions for potential communication links with other nodes. The sensor is represented at the center of the figure, and solid shading indicates that this sensor is currently active. The arrows emanating from the node indicate the darts, showing actual (thicker solid lines) and potential (thin hatch strokes) communication routes, which serve as edges of the triangulation. The length of the arrows represents the qualitative distance to its partner node. The ordering of darts around the node corresponds to the relative qualitative direction of each neighboring node.

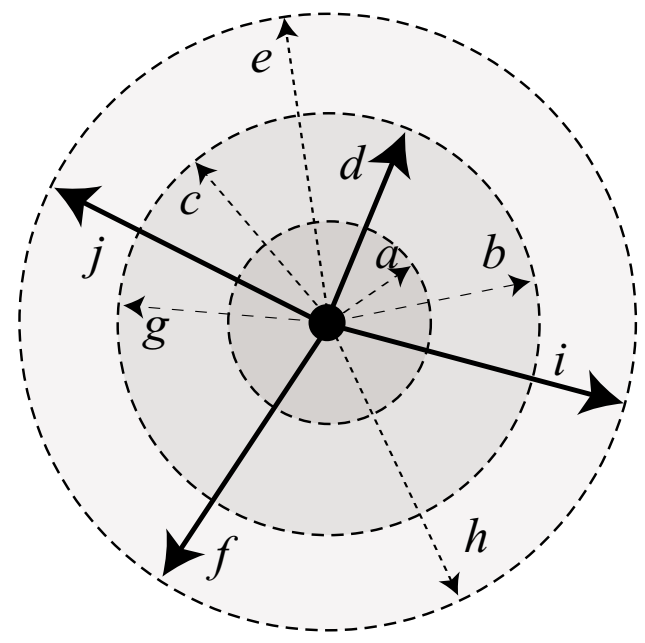

Figure 3: Schematic of sensor vertex

After the sensors have been deployed, the network is initialized. In the initialization phase, a small proportion of sensors will be designated active, and active sensors will negotiate appropriate communication partners. An algorithm for building and maintaining the communication network is described in section 5 . In the active network phase, the sensor network is required to respond to qualitative changes in the dynamic field (see Figure 2). As the field varies with time the sensors have a number of tasks:

- data collection for active sensors;

- data and control communication between neighboring active sensors; and

- decisions about activating and deactivating themselves, or neighboring sensors.

These decisions are based on changes to field values and communication with neighboring nodes. For example, a denser configuration of sensor node communication might be required at a location where a qualitative change to a region of activity is being detected. We note that all such decisions are local (made by the sensors and their neighbors) and based only upon qualitative information. Figure 4 shows a small subset of sensors in an imaginary configuration. There are four active sensors, and two inactive sensors, indicated by black and white centers, respectively. Full and hatched strokes indicate active and potential communication links, respectively. Each node stores its local part of the combinatorial map.

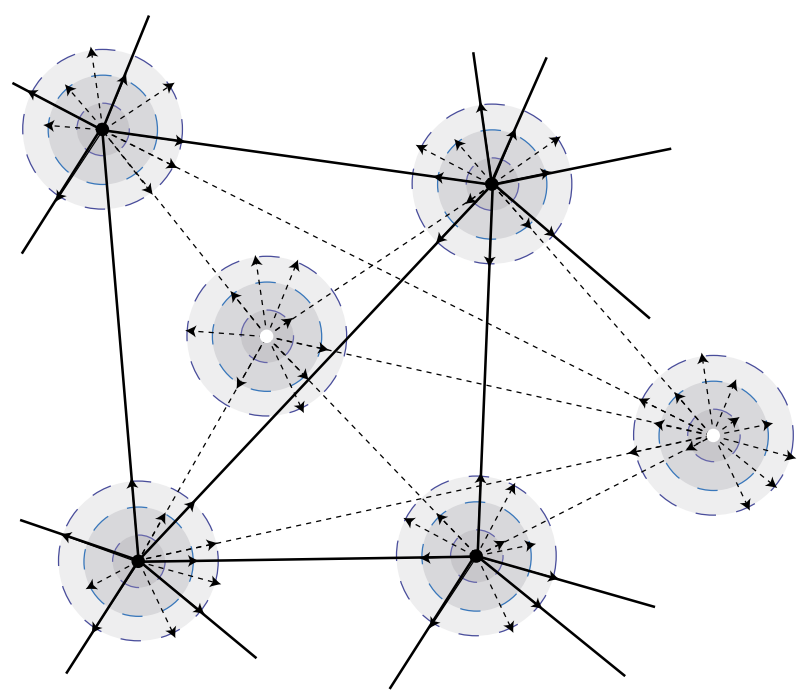

Figure 4: Small subset of a sensor configuration

\section{SENSOR NETWORKS}

\subsection{Limitations of Sensor Networks}

The challenge in building the communication topology occurs when establishing which neighboring nodes based on the combinatorial map should be selected by a node with the objective to create a robust communication network. The choice is limited by the following constraints:

- The link quality between a sensor node and its neighbors is often asymmetric and varies over time [24].

- Sensor nodes might be in sleep mode, busy and/or low on battery supply, and not respond to requests. Idle listening of nodes is an additional battery drain.

- Once a communication link is established, this link is prone to failure over time and needs to be checked repeatedly.

- The exact geographic location of sensors is often not known.

- Communication cost is a significantly higher drain on the battery than computation; thus, messages and message size needs to be minimized. 


\subsection{Qualitative In-Network Data Processing}

Today, data processing for sensor networks is based on quantitative assessment of dynamic fields. SQL-like queries, i.e. "Report the average temperature over a certain region" use in-network data aggregation such as average, maximum or minimum values [10]. More holistic aggregate functions allow to estimate regions of dynamic spatial fields [23]. In principle, all measured values are passed between the nodes in the network, computed, aggregated, and passed on through the network to create the final results. On the other hand, the execution of the proposed qualitative queries is significantly different. Here, values are assessed locally, and only compared between neighboring nodes.

The following section describes an algorithm for building and maintaining an adaptive sensor network capable of responding to changes in its sensing environment in a qualitative way. The approach is designed to allow for the inherent limitations of sensor networks in several ways.

We note that our geosensor network model does not assume that exact or absolute geographic location is known for nodes. Instead, we do assume the sensors are able to determine the relative qualitative direction of nearby sensors needed in order to construct the cyclic ordering of darts required for the combinatorial map. Clearly, such information can easily be generated in cases where absolute geographic location is known, but may also be sensed indirectly using direction finding techniques, such as acoustic direction finding (see, for example, $[3,14]$ ). In addition, when precise measurements bay not be available, it may be possible to derive qualitative knowledge of the relative distances of nearby sensors (e.g., close, midrange, far), for example based on wireless signal strength. The simplest case is to assume that sensor nodes know their own geographic location, and broadcast it and their sensor node ID during an initialization phase to establish the combinatorial map.

A variety of further advantages to the approach are expected, although at this point further work is needed to verify empirically these expectations:

- Battery drain is minimized by allowing the sensors to go into a sleep mode when the sensor's local environment is stable or otherwise not relevant to a query. The geosensor network is effectively able to "zoom in" on specific areas of interest, "zooming out" again when a region becomes stable once more.

- Where there is excess sensing capacity (i.e., the network is zoomed out), sensors may be excluded from the network based on a variety of factors including remaining battery life. Thus, the sensor network may "save" sensors nearing the end of their battery life in case they are subsequently needed to refine the sensor network granularity.

- The sensor network's ability to continually adapt to changes in the sensed environment, also enables it to respond to changes in the network environment, primarily loss of connectivity.

- The focus on local communication and in-network query processing means that the communication overheads of the sensor network are much lower, with less frequent requirements to flood the network with information.
- Sending only qualitative information about change between sensor nodes minimizes the communication in several ways: qualitative information can be reduced to "change happened" or to "small change" or "significant change" happened. This information can be represented in by one or two bits; thus, message size and data packets are kept very small and constant. Information is also only passed between neighbors.

\section{GEO-RESPONSIVE SENSOR NET- WORK ALGORITHM}

As described previously, the communication strategy between sensor nodes in the network is based on a triangulation. This allows the creation of a communication topology within the sensor network that is appropriate for qualitative change detection. In this section we describe the algorithm for the creation of a triangulation in the sensor network and adaptation of this triangulation in response to changes in the dynamic field. In this section, we detail an algorithm to establish such a geo-responsive sensor network once the nodes are deployed and activated in the geographic environment.

We assume that each geosensor can detect and store relative signal direction of other geosensors it can hear based on the sensor node's ID and location (section 4). It also orders all potential neighbors based on these relative bearings. Each geosensor node can be active (processing, communicating) or inactive (sleep mode) in the geo-responsive sensor network. A geosensor can activate itself either by detecting a significant change in its environment during periodic wake-up cycles, or receiving a request from a neighboring geosensor.

The central idea for building, refining, and coarsening the communication topology of the geosensor network is that we can only add and remove sensors in such a way that individual triangles in the triangulation are added or removed. Thus, when adding or removing triangles, a number of neighboring nodes may need to communicate to coordinate their de/activation. Crucially:

- only local communication is required with sensors that are in direct communications range of each other; and

- activation or deactivation only happen around regions of significant change or stability, and not throughout the entire network.

\subsection{Activating sensors}

When activating sensors there are four cases to consider.

\section{Activation case 1}

This case applies when a particular node is inactive and needs to activate itself, but it is not in communication range of any active node. In this case, to activate the node requires two neighboring inactive nodes which are in range of each other, but also not in range of any other active nodes. This effectively creates a new disconnected triangle of communication in our (possibly empty) triangulation. Figure 5 illustrates case 1 , where nodes $i, j$, and $b$ have formed a triangle.

During activation, the nodes must communicate to coordinate their activation. Coordinated activation can be achieved using limited communication resources by means of an activation message. For example, in figure 5 node $i$ 
broadcasts an activation request. Any nodes within communication range (in our example, $a, b, f, g, h, k, j$ ) may respond to this request. In this case, node $j$ responds by adding its own (participation) information to the message, and broadcasts it to all nodes in its own communication range. Any of the nodes who receive this message except for the originating node (in this case $b, c, d, e, f, k$ ) may append their own information and send the message only back to the first listed node (in this case $i$ ). In figure 5 node $b$ does this. To confirm the activated link, the completed message must be passed round the triangle a second and final time.

In the case that there is more than one possible nodetriangles that respond to a node's request for activation (e.g., perhaps $i h g, i k a, i j g$ also responded to $i$ 's activation request), the initiating node is responsible for selecting one of the possible triangles to activate. This decision may be based on a number of factors, such as signal strength outlined previously, or distance of the node from the originating node. Whatever criteria are used to decide between multiple possibilities for this and all subsequent cases activation is an atomic transaction, to ensure the triangulation is wellformed. As soon as a node forwards a completed message (indicating activation confirmation), it is unable to forward any other activation confirmations until it has received its final confirmation response. A timestamp on the completed activation message is used to allow triangle activation to timeout in cases where network or geosensor outage means activation is not completed.

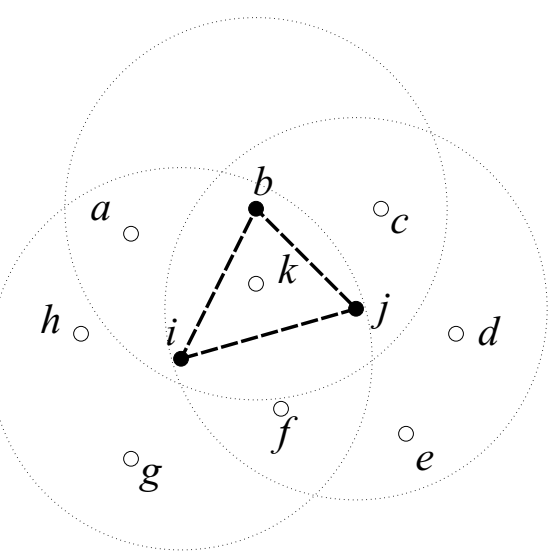

Figure 5: Activation case 1

\section{Activation case 2}

This case applies when a particular node is inactive and needs to activate itself, but is in range of at least two active nodes. Then we have two subcases:

Activation case 2.1 If the candidate for activation is inside a triangle of active nodes, then we need to subtriangulate that triangle. This is done by establishing links between the new node, and its three active neighbors. Figure 6 illustrates this case. Note that we need the ordering information about the relative direction of neighbors around a node to verify that a candidate node is indeed inside a particular triangle. The combinatorial map information is necessary to decide whether the candidate for activation is inside a particular triangle or not.

For example, to add the new node $k$, the counterclockwise ordering of $k$ must be between $j$ and $b$ from $i$, between $b$ and $i$ from $j$, and between $i$ and $j$ from $b$. As for activation case 1 , it may be necessary to establish some criteria, such as remaining battery life, for choosing between multiple nodes competing to establish themselves inside the same triangle.

Activation case 2.2 If the candidate for activation is outside a triangle of active nodes, then we need to append a new triangle to the edge of the triangulation. Again, using the ordering information in the combinatorial map enables the relative position of the candidate node to be efficiently determined. Figure 7 illustrates this case. Similar to activation case 1 , the node needing to establish a communication triangle (node $f$ ) broadcasts an activation request. In this example, active nodes $i$ and $j$ respond. Again, $i$ and $j$ must check the ordering of directions for $f$ from the combinatorial map (the counterclockwise ordering of $f$ must be between $b$ and $j$ from $i$ and between $i$ and $b$ from $j$ ).

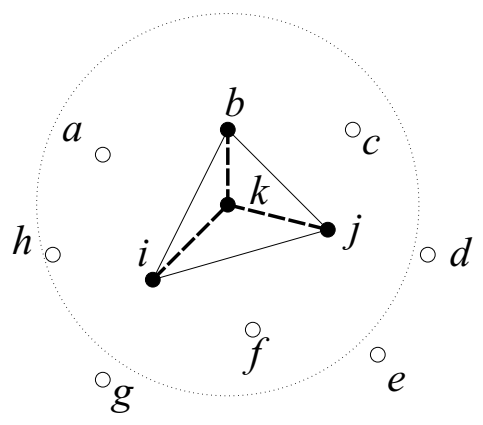

Figure 6: Activation case 2.1

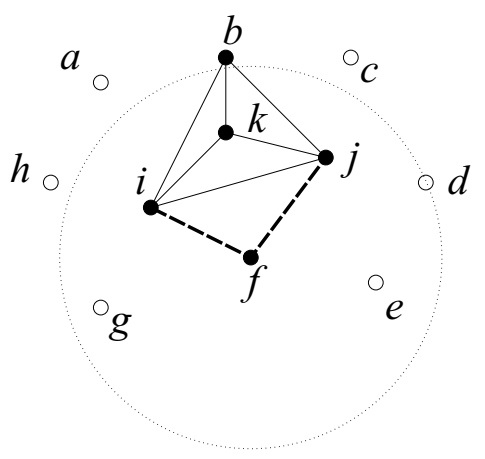

Figure 7: Activation case 2.2

\section{Activation case 3}

An optional case occurs when a node is already active and is in communication range of another node with which it is not actively linked. In such a case it may be desirable for such an active nodes to link up by adding a triangle at the edge of a triangulation. Figure 8 illustrates this case, with the thick dashed line showing the potential to add a new 
link between two already active nodes $(a$ and $b$ ). Such link activation extends the spatial coverage of the monitoring network, i.e., a new triangle is added to the sensor network for which we can interpolate sensed information.

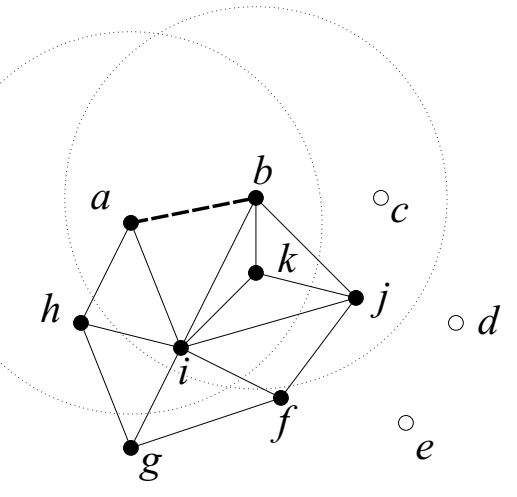

Figure 8: Activation case 3

\section{Activation case 4}

If any conditions other than those listed above apply, then the node is barred from activating itself to prevent invalid triangulations. However, such a sensor may still forward to its immediate neighbors a request for activation. Neighboring nodes who receive this request can respond by attempting to activate. Assuming these nodes satisfy at least one of the activation cases, then their own activation will effect changes in the immediate network environment of the original node, which in turn may enable it to activate itself.

\subsection{Deactivating sensors}

The principles behind deactivating sensors can be the same as for activating sensors. Thus, four deactivation cases can be constructed as the converse of the four activation cases. In such cases, a neighboring node may block deactivation of a triangle of nodes if changes in its vicinity warrant it. However, this assumes sensors of a triangle only deactivate as an atomic operation in response to changes in the sensed values in their immediate environment or requests from other sensors. In practice, sensors often lose network connection as a result of link failure, hardware failure, power failure, or some other factor such as interference. Hence, we must also have strategies to deal with link failures.

\subsection{Dealing with link failure}

We require another deactivation case when a sensor becomes inaccessible to the network for whatever reason. Effectively any sensor has an autonomous veto over participation in the network. Where such deactivation occurs, the loss of connectivity with be detected by neighboring active nodes. If attempts to re-establish the connection fail, all links uniquely associated with a triangle containing the deactivated node must be terminated to preserve the triangulation. Further, any nodes that become isolated as a result of these changes must deactivate. However, these nodes are free to attempt reactivation in cooperation with other neighboring nodes. Figure 9 illustrates deactivation due to link failure, with the effects of an enforced deactivation (node $i$, indicated by a white circle) leading to the 11 links being deleted (dashed lines) and three further nodes being deactivated ( $a, h, g$, indicated by gray circles). Note that the effects of link failure to not propagate throughout the sensor network, and are confined nodes in the communication region of the node that fails.

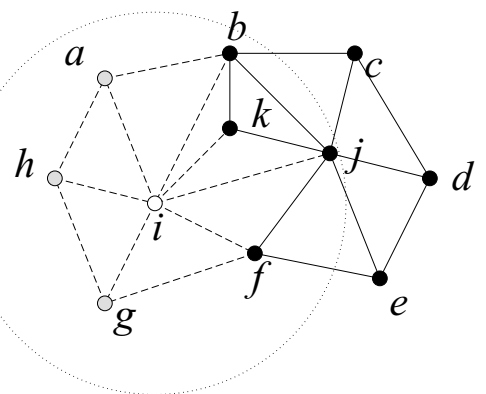

Figure 9: Deactivation due to link failure

\subsection{Properties of the georesponsive sensor net- work}

The algorithm described above has three important properties, summarized below:

1. The network triangulation always succeeds, but may not be connected. Since each atomic action can only add or remove a complete triangle to the triangulation, changes preserve the basic triangulated structure. However, the triangulation may not necessarily be connected. Individual triangulations can grow from multiple start triangles, and can coalesce or fragment.

2. Nodes can activate or deactivate in response to changing sensed values for their local environment. When stable, sensors can drop out of the communication network in order to preserve battery power

3. The algorithm for building and adapting the set of triangulations is local, and for each node only requires communication with and knowledge of its immediate neighbors.

\section{QUERY PROCESSING USING A GEO- RESPONSIVE SENSOR NETWORK}

The previous section described the algorithm for building the triangulated communication network and enabling that network to adapt to local conditions. As the creation of an initial map forms a triangulation over the entire or parts of the network, the data or query processing topology is thereby established. Once a query is inserted in to the geosensor network, it is flooded to all sensor nodes and stored locally at each node. Nodes that subsequently become active in the network, following a request from a nearby active node, will be updated with the current query when they activate. As an example, we assume the georesponsive sensor network is tasked with responding to a single query, such as "Track all emergent toxic cloud fields, report their boundary, and report any formation of holes, over the next 2 months." Concurrently executing multiple queries in the same network will be the subject of further work. 
When a query is inserted into the sensor network, all nodes store the query's timestamp, the query predicates (i.e. "detect emergence" and "define boundary"), as well as the reporting interval. The nodes start a continuous query execution by sensing locally. When an inactive node during one of its periodic wake-up periods detects significant changes, it attempts to activate by joining in a triangulation with nearby active sensors or initiating an active triangulation (see section 5). Conversely, when an active node senses local stability, it will attempt to deactivate itself and the triangles it belongs to. The active nodes must occasionally communicate with nodes in their triangles periodically waking up in cycles to check that each triangle is still "alive." The wake-up frequencies are aligned with the dynamic spatial field type or application area for which the sensor network is deployed.

We assume that a sensor node has either built-in knowledge of "significant" local change and/or are initialized by a continuous query as described above. Initially, geosensors measure in well-defined intervals, and only locally compare the measured values with their predefined range of acceptable values, and/or historical values. This avoids any communication until change is detected. Once a sensor node detects change, it starts communicating with neighboring nodes to assess the spatial extent and significance of the change in its neighborhood. It is crucial that the map creation and adaptation is tightly integrated with the data processing task.

Two key classes of query to which the geosensor network must be able to respond correspond to our two classes of emergent qualitative entities: continuants and occurrents. We refer to these query types as tracking and event queries, respectively.

\subsection{Tracking queries}

Tracking queries concern tracking entities such as regions, boundaries, and holes. As an example, in order to determine the extent of a particular region at any moment, the sensor network must find those sensors which are at the boundary of that region. A sensor can detect locally whether it is at the boundary of a region simply by querying its immediate neighbor nodes. For example, consider an active node that has detected a high concentration of an airborne toxin in its immediate vicinity. If any of its immediate (active) neighbors have not detected this high concentration, then the node must be at the boundary of the toxic cloud. (This is a simplification, as it assumes perfect sensor accuracy/precision as well as crisp values for the field. See the next section for extensions to this work.) In fact, if we call such a sensor a "boundary-plus node," we might also define the dual concept of a "boundary-minus" node, which has not detected the toxin but has at least one neighbor that has. In a similar way we might also define the interior and exterior nodes with respect to the toxic cloud.

Boundary nodes then discover the current state of the boundary of a region by locally checking which of its immediate neighbors is also a boundary node. Figure 10 illustrates the concept, where the grey cloud represents a spatial field, such as toxic gas cloud; inactive sensors are shown as white nodes; active sensors are shown as black nodes; those active sensors which have detected they are part of the boundary of the cloud are shown with a thick white annulus. Assuming the temporal granularity of the individual sensors is suffi- ciently high, the sensors can track dynamic changes in the field. Current research by the authors is addressing formalizing these simple notions of sensed regions and boundary, for example by formally defining topological regularity for such regions.

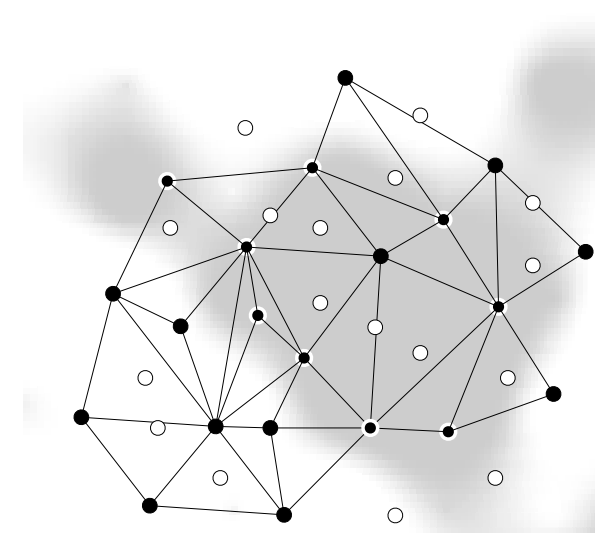

Figure 10: Tracking the boundary of a region of a field

\subsection{Event queries}

Event queries concern the identification of events that occur in the dynamic field, such as the appearance or disappearance of regions and holes. For the toxic cloud, the appearance of a region can be detected locally by a sensor as a change from not sensing the toxin to sensing the toxin, while neighboring nodes continue not to detect the toxin. Similarly, the appearance of a hole in can be detected locally by a sensor as a change from being an interior node (surrounded only by nodes that have also detected the toxin) to an exterior node (no longer detecting the toxin) whilst all the nodes neighbors continue to detect the toxin. Current research by the authors is formalizing these notions using conceptual neighborhoods between node states. Using this approach, it is possible to classify a range of different events which can be detected using local rules, including appearance and disappearance of regions and holes, as well as forms of movement. With the addition of definitions of regularity, further events can be discerned, such as region splitting and fusion.

\section{CONCLUSIONS}

The research reported in this paper has the aim of providing mechanisms for networks of sensors embedded in a geographic space to be effective in response to dynamic geographic phenomena. We have focused specifically on utilizing the qualitative aspects of measurements and salient features of the phenomena under investigation to achieve efficiency gains in network responsiveness. The formal framework around which our approach has been built is the notion of a combinatorial map, which provides the ability to deduce global characteristics of the space from local properties at vertices of the map. Geometrical constraints are introduced using surface triangulations. The key to this work is that it provides mechanisms by which the network can dynamically auto-configure in response to changes in the dynamic field, and also in response to the changing nature of query types. 
The team is currently involved in a larger-scale simulation for testing these ideas.

We have said very little about the precise nature of the triangulation. Of course, given a set of points on a surface, there are many different triangulations possible. One important area of future work is to develop triangulations that more efficiently triangulate the environment. Delaunay triangulations and their duals, Voronoi diagrams, have been the subject of considerable research (see, for example $[2,20]$ ) and would clearly be ideal for ensuring a sensor network triangulation with desirable properties. There has also been work that allows Delaunay triangulations to be "dynamic" in the sense of capable of reconfiguration as vertices move or change (e.g., $[15,16])$, which may prove useful in maintaining a Delaunay triangulation in the face of activation and deactivation of nodes.

Another important area for future work is the introduction of uncertainty. There are several ways in which uncertainty enters into this research. First, the sensors themselves may have various uncertainty dimensions, such as unreliability, imprecision, and inaccuracy of measurements. Second, the fields under investigation may not be crisp. For example, if we are looking for dangerous levels of toxicity in a gaseous chemical, there will be a gradation linked to quantitative elements such as density, temperature, etc. Approaches from fuzzy analysis will need to be incorporate to take account of these factors.

Overall, introducing qualitative and topological elements to geo-sensor networks holds out considerable hope for their more effective deployment. This paper sets out some of the early stages in this approach.

\section{REFERENCES}

[1] S. Ahmad and S. P. Simonovic. Spatial system dynamics: New approach for simulation of water resources systems. Journal of Computing in Civil Engineering, 18(4):331-340, 2004.

[2] F. Aurenhammer. Voronoi diagrams: A survey of a fundamental geometric data structure. $A C M$ Computer Surveys, 23:345-405, 1991.

[3] J. C. Chen, J. Yip, L.and Elson, H. Wang, D. Maniezzo, R. E. Hudson, K. Yao, and D. Estrin. Coherent acoustic array processing and localization on wireless sensor network. Proceedings of the IEEE, 91(8), 2003.

[4] Jean-Francois Dufourd and Francois Puitg. Functional specification and prototyping with oriented combinatorial maps. Comput. Geom. Theory Appl., 16(2):129-156, 2000.

[5] J.R. Edmonds. A combinatorial representation for polyhedral surfaces. Notices Amer. Math. Soc., 7:646, 1960.

[6] A. Galton. Qualitative Spatial Change. Oxford University Press, Oxford, England, 2000.

[7] A. Galton. Fields and objects in space, time, and space-time. Spatial Cognition and Computation, 2004. forthcoming.

[8] P. Grenon and B. Smith. SNAP and SPAN: Towards dynamic spatial ontology. Spatial Cognition and Computation, 4(1):69-104, 2004.

[9] L. Guibas and J. Stolfi. Primitives for the manipulation of general subdivisions and the computation of voronoi. ACM Trans. Graph., 4(2):74-123, 1985.

[10] J. Hellerstein, W. Hong, S. Madden, and k Stanek. Beyond average: Towards sophisticated sensing with queries. In IPSN'03, 2003.

[11] C. Jensen. Database aspects of location-based services. In J. Schiller and A. Voisard, editors, Location-Based Services, chapter 5, pages 115-147. Morgan Kaufmann, 2004.

[12] J. G. Kroes, J. G. Wesseling, and J. C. Van Dam. Integrated modelling of the soil-water-atmosphere-plant system using the model SWAP 2.0: An overview of theory and an application. Hydrological Processes, 14(11-12):1993-2002, 2000.

[13] D. Livingstone, J. Raper, and T. McCarthy. Integrating aerial videography and digital photography with terrain modelling: An application for coastal geomorphology. Geomorphology, 29(1-2):77-92, 1999.

[14] R. L. Moses, D. Krishnamurthy, and R. M. Patterson. A self-localization method forwireless sensor networks. EURASIPJournal on Applied Signal Processing, 4:348-358, 2002.

[15] M.A. Mostafavi and C. Gold. A global kinetic spatial data structure for marine simulation. International Journal of Geographic Information Science, 18(3), 2004.

[16] M.A. Mostafavi, C. Gold, and M. Dakowicz. Delete and insert operations in Voronoi/Delaunay methods and applications. Computers and Geosciences, 29(523-530), 2003

[17] R. Muetzelfeldt and M. Duckham. Dynamic spatial modeling in the Similie visual modeling environment. In P. F. Fisher and D. J. Unwin, editors, Re-presenting GIS, chapter 17, pages 244-256. John Wiley, 2005.

[18] S. Nittel, M. Duckham, and L. Kulik. Information dissemination in mobile ad-hoc geosensor networks. In M.J. Egenhofer, C. Freksa, and H.J. Miller, editors, GIScience 2004, volume 3234 of Lecture Notes in Computer Science, pages 206-222. Springer, Berlin, 2004. (Conference accepted $35 \%$ of submitted papers).

[19] S. Nittel, A. Stefanidis, I. Cruz, M. Egenhofer, D. Goldin, A. Howard, A. Labrinidis, S. Madden, A. Voisard, and M. Worboys. Report from the first workshop on geo sensor networks. ACM SIGMOD Record, 33(1), 2004.

[20] A. Okabe, B. Boots, and K. Sugihara. Spatial Tessellations: Concepts and Applications of Voronoi Diagrams. Wiley, Chichester, UK, 2nd edition, 2000.

[21] D. Peuquet. Making space for time: Issues in space-time data representation. Geoinformatica, 5(1):11-32, 2001.

[22] J. Raper and D. Livingstone. Development of a geomorphological spatial model using object-oriented design. International Journal of Geographic information Systems, 9(4):359-184, 1995.

[23] M. Sharifzadeh and C. Shahabi. Supporting spatial aggregation in sensor network databases. In $A C M-G I S$ Conference, Washington, D.C., 2004.

[24] X. Wang, G. Xing, Y. Zhang, C. Lu, R. Pless, and C. Gill. Integrated coverage and connectivity configuration in wireless sensor networks. In First ACM Sensys Conference, Los Angeles, CA, 2003. 
[25] O. Wolfson, B. Xu, S. Chamberlain, and L. Jiang. Moving objects databases: Issues and solutions. In Proc. 10th International Conference on Scientific and Statistical Database Management (SSDBM), pages 111-122. IEEE Computer Society, Washington, DC, 1998. 


\section{University Library}

- MIN E R VA A gateway to Melbourne's research publications

Minerva Access is the Institutional Repository of The University of Melbourne

Author/s:

DUCKHAM, M.; NITTEL, S.; WORBOYS, M.

Title:

Monitoring dynamic spatial fields using responsive geosensor networks

Date:

2005

Citation:

Duckham, M., Nittel, S., \& Worboys, M. (2005) Monitoring dynamic spatial fields using responsive geosensor networks. In, Proceedings, ACM GIS 2005, Bremen, Germany.

Publication Status:

Published

Persistent Link:

http://hdl.handle.net/11343/34961

File Description:

Monitoring dynamic spatial fields using responsive geosensor networks 


\section{University Library}

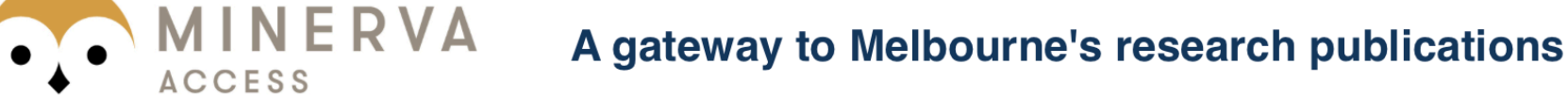

Minerva Access is the Institutional Repository of The University of Melbourne

Author/s:

Duckham, M;Nittel, S;Worboys, M

Title:

Monitoring dynamic spatial fields using responsive geosensor networks

Date:

2005-12-01

Citation:

Duckham, M., Nittel, S. \& Worboys, M. (2005). Monitoring dynamic spatial fields using responsive geosensor networks. GIS: Proceedings of the ACM International Symposium on Advances in Geographic Information Systems, pp.51-60. ACM. https:// doi.org/10.1145/1097064.1097073.

Persistent Link:

http://hdl.handle.net/11343/30043 